\title{
Bilateral dyshidrosiform pemphigus vulgaris: an unusual clinical presentation
}

\section{Introduction}

Pemphigus vulgaris is an autoimmune bullous disease clinically characterized by flaccid cutaneous blisters and erosions associated to mucosal involvement. ${ }^{1}$ In addition to the cutaneous and mucosal surfaces, the pharynx, vocal folds, and anogenital mucosa may also be affected. ${ }^{2}$ Unilateral or localized forms of pemphigus have been reported in literature. Unlike dyshidrosiform pemphigoid, which is a well-known variant of localized pemphigoid, dyshidrosiform pemphigus remains very rare. ${ }^{1}$

We report a case of a 59 year old man, with isolated bilateral large plantar erosion associated to pompholyx like lesions.

\section{Case report}

A 59-year-old man, chronic smoker, presented to our departement with bilateral painful plantar erosions, overflowing on the lateral sides of the feet with some plantar vesicles and pustules, evolving since 5 months (Figure 1). Nail anomalies of the toes were also noted, like perionyxis and onychodystrophy. He reported having chronic oral and genital erosions that have progressed over the past 10 years in flareups and incomplete remissions. There was no lesion elsewhere in the body. Two weeks later, he developed several annular bullous necrotic lesions on the back with some pseudococards lesions (Figure 2) with extension of oral and genital erosions (Figure 3).

Tzanck test of lesions of oral mucosa showed acantholytic cells. Histopathology was suggestive of pemphigus vulgaris. Elisa showed positive anti-desmoglein 1 at 360 UI and anti-desmoglein 3 at 800 UI. The diagnosis of dyshidrosiform pemphigus vulgaris was confirmed. He was treated with prednisolone $\mathrm{mg} / \mathrm{kg} /$ day $(60 \mathrm{mg} /$ day) and subsequently with rituximab $1 \mathrm{~g}$ and 3 sessions of plasmapheresis, with very good improvement with complete healing of plantar lesions (Figure 4). The patient was subsequently declared covid 19 positive and the 2nd rituximab perfusion was postponed.

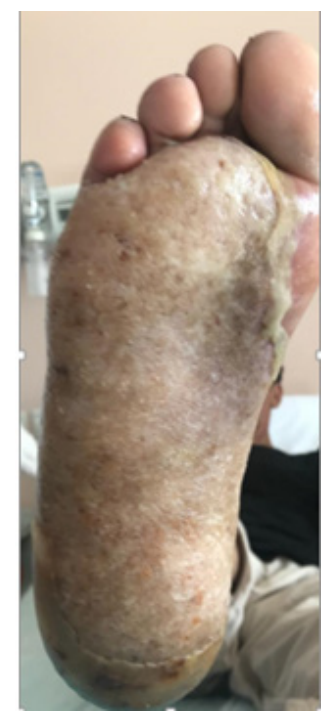

Figure I Painful plantar erosions, with some plantar vesicles and pustules.

\author{
Volume 5 Issue 2 - 202I
}

\begin{abstract}
A Khouna,' H Ragragui,' M Benkaraach,' N Zizi, $^{1,2}$ S Dikhaye ${ }^{\prime, 2}$

'Department of Dermatology, Mohammed VI University Hospital of Oujda, Medical School of Oujda, Mohammed First University of Oujda, Morocco

${ }^{2}$ Epidemiology, Clinical Research and Public Health Laboratory, Medical School of Oujda, Mohammed First University of Oujda, Morocco
\end{abstract}

Correspondence: Afaf Khouna, Department of Dermatology, Mohammed VI University Hospital of Oujda, Medical School of Oujda, Mohammed First University of Oujda, Morocco, Tel+2I2+664826702, Email afaf.khouna@gmail.com

Received: April 14,2021 | Published: April 27, 2021

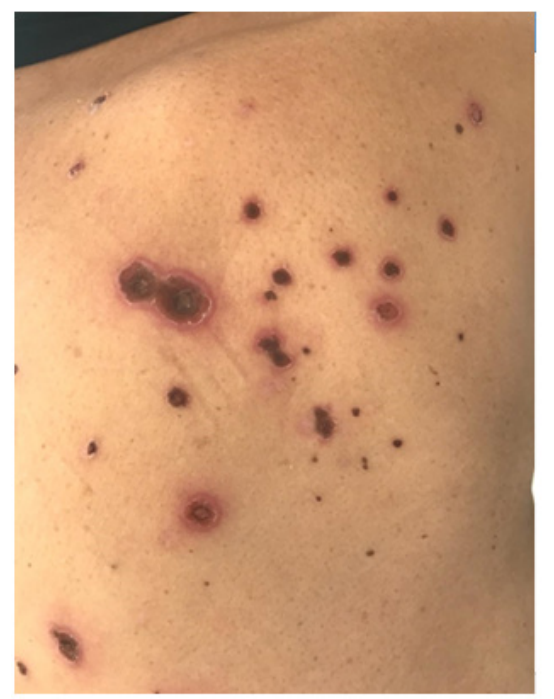

Figure 2 Annular bullous necrotic lesions on the back with some pseudococards lesions.

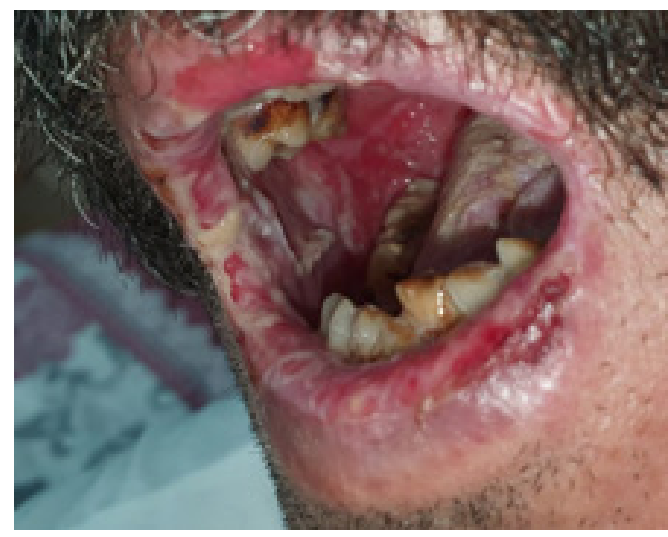

Figure 3 Oral erosions. 


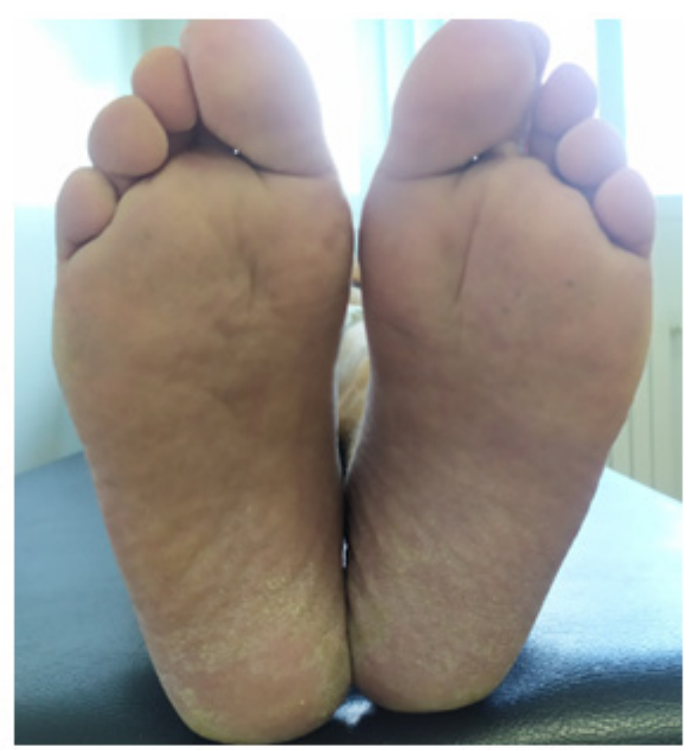

Figure 4 Complete healing of plantar lesions.

\section{Discussion}

Some rare presentations of pemphigus vulgaris have been reported, including isolated esophageal involvement, recurrent paronychia, conjunctival mass, foot ulcers, discoloration of toes with hyperkeratotic skin lesions and verrucous lesions. ${ }^{1,3}$ Palmoplantar involvement in pemphigus vulgaris is a poor prognostic indicator. Dyshidrosiform pemphigus vulgaris is an unusual presentation which led to a delay in diagnosis. Unlike dyshidrosiform pemphigus, dyshidrosiform pemphigoide is a well-known variant of localized pemphigoid presenting as vesiculobullous lesions on palms and soles. ${ }^{1}$ Usually, an autoimmune bullous disease, is not initially considered in patients who present with blisters restricted to the palms and/or soles. Indeed, this dyshidrosiform bullous mimics several other conditions, such as allergic and irritant contact dermatitis, chronic bullous disease of childhood, cutaneous T-cell lymphoma, dermatophyte infection, dyshidrosis or pompholyx, epidermolysis bullosa acquisita, erythema multiforme, herpes gestationis, lichen planus, linear IgA disease, scabies, and systemic contact dermatitis. ${ }^{4}$ Painful plantar erosions can be also seen in erosive plantar lichen planus which is a rare. Nail involvement in pemphigus, including atrophy of the nail bed, dorsal pterygium formation, and toenail loss with scarring, has also been reported. ${ }^{5}$

The presentation in our patient was unusual as the disease started as pompholyx like plantar lesions with painful large erosions.
Other diagnosis was considered in our patient such as infected dyshidrosiform eczema and bullous erythema multiforme. The oral and genital lésions and the late bullous lesions of the back directed us towards an autoimmune bullous dermatosis. Pemphigus vulagris was confirmed on Tzanck test, histopathology and Elisa.

Exact pathogenesis of dyshidrosiform pemphigus is unknown. However a hypothesis of dyshidrosiform pemphigoid was proposed incriminating local inflammatory process which can uncover the masked antigen and trigger the disease at local site. ${ }^{1}$

This unusual presentation can lead to a delay in diagnosis and proper treatment. Hence, we present our case to highlight a rare morphologic variant of pemphigus.

\section{Conclusion}

Despite it is a very rare presentation, auto-immune diseases should be kept in mind in cases with pompholyx like lesions and palmoplantar erosions. Dyshidrosiform bullous pemphigoid is well known, however dyshidrosiform pemphigus vulgaris remains rare.

\section{Conflicts of interest}

There are no conflicts of interest.

\section{Acknowledgments}

None.

\section{Funding}

None.

\section{References}

1. Daroach, Manju De, Dipankar Handa, et al. Unilateral Dyshidrosiform Pemphigus Vulgaris. Annals of the National Academy of Medical Sciences (India). 2020;56:231-233.

2. Di Lernia V, Casanova DM, Goldust M, et al.Pemphigus Vulgaris and Bullous Pemphigoid: Update on Diagnosis and Treatment. Dermatol Pract Concept. 2020;10(3):e2020050.

3. H Benhiba, S Hamada, N Guerouaz, et al. Pemphigus vulgaire de présentation inhabituelle, Annales de Dermatologie et de Vénéréologie. 2013;140(2):116-119.

4. Cohen PR. Dyshidrosiform Bullous Pemphigoid: Case Reports and Review. Cureus. 2020;12(1):e6630.

5. Cohen, Brandon Madu, Pamela Smogorzewski, et al. Painful plantar erosions. International Journal of Dermatology. 2020;59(11):1341-1342. 